\title{
DNA fragmentation index and its correlation with other sperm parameters in 4833 infertile males
}

\author{
Priya S. Patil ${ }^{1 *}$, Satish M. Patki ${ }^{2}$, Shweta S. Patki ${ }^{3}$, Ujjwala S. Patki ${ }^{3}$
}

\begin{abstract}
${ }^{1}$ Department of Anatomy, Bharati Vidyapeeth Medical College and Hospital, Sangli, Maharashtra, India
${ }^{2}$ Department of Gynaecology, D. Y. Patil Education Society, Kolhapur, Maharashtra, India

${ }^{3}$ Department of Gynaecology, Patki Hospital and Research centre, Kolhapur, Maharashtra, India
\end{abstract}

Received: 02 July 2018

Accepted: 27 July 2018

*Correspondence:

Dr. Priya S. Patil,

E-mail: drpriyapatil22@gmail.com

Copyright: (c) the author(s), publisher and licensee Medip Academy. This is an open-access article distributed under the terms of the Creative Commons Attribution Non-Commercial License, which permits unrestricted non-commercial use, distribution, and reproduction in any medium, provided the original work is properly cited.

\section{ABSTRACT}

Background: Semen analysis forms the basic investigation for male factor of infertility. According to WHO Manual sperm values of normalcy are decreasing. Recent studies have highlighted newer parameters of sperm playing their role in the outcome of pregnancy. The aim was to study the DNA fragmentation index (DFI) and to know its correlation with other sperm parameters.

Methods: A total of 4833 cases underwent routine semen analysis along with the DFI. The statistical analysis was done using SPSS using t test and ANOVA test.

Results: It was observed that $52 \%$ infertile males presented late for treatment. All parameters showed a decline with advancing age. There was definite correlation between sperm count and sperm motility as well as morphology. The DFI showed significant negative correlation with sperm count. Pearson correlation showed positive correlation between DFI and sperm motility and morphology.

Conclusions: The study highlights the need for increasing awareness about male factor of infertility. A thorough semen analysis along with DFI can help the clinician to explain the couple in a better way about the choice of ART and success of treatment.

Keywords: DFI, Progressive sperm motility, Sperm concentration, Sperm morphology

\section{INTRODUCTION}

Reproductive biology is advancing at an enormous pace, yet about 60-80 million couples face problems of fertility worldwide and the infertility rate is about 8 to $12 \%^{1,2}$

Out of the total number of cases $40 \%$ of infertility cases are related to the male factor. ${ }^{3}$ Semen analysis forms the basic investigation for male infertility.

Recent advances in infertility management have highlighted newer parameters like sperm morphology and sperm chromatin integrity playing their role in the outcome of pregnancy. ${ }^{4-6}$ An infertile couple is offered treatment in the form of many different assisted reproduction techniques (ART). ${ }^{4}$ Considering the above facts there is a need for large population-based studies to know the range of routine sperm parameters.

The present prospective study was conducted to know the sperm parameters with emphasis on sperm concentration, sperm motility and DNA fragmentation index (DFI). The correlation of DFI with other sperm parameters is also studied. Further we discuss the impact of these parameters on infertility management with its implications on choice and results of ART. 


\section{METHODS}

The study was approved by the Institutional Ethics Committee and informed consent was obtained from all the patients. The study was an observational, prospective and laboratory based during a period of two years, from May 2014 to May 2016. During this period, we examined 5216 men attending infertility clinic.

\section{Exclusion criteria}

- 383 men had azoospermia

- The subjects with a history of smoking, chewing tobacco, alcohol consumption, orchitis, varicocele, tuberculosis, diabetes mellitus, hypertension and job stress.

- The patients who suffered from viral/bacterial infection in the previous 4 weeks.

- Those with a history of cardiac, neurological or nephrotic disease

- Those who had a family history of any genetic disease.

\section{Sample size}

Considering that the incidence of infertility is 8 to $12 \%$ the sample size was calculated. ${ }^{1}$ For $8 \%$ incidence the sample size was 4600 and for $12 \%$ incidence it was 2933.

The total cases of male infertility in the study period were 5216, out of which those having azoospermia were 383 which were excluded. So, the sample size was 4833 .

A total of 4833 cases underwent routine semen analysis, performed according to the WHO 2010 Manual. ${ }^{5}$ The protocols described in WHO laboratory manual for the examination and processing of human semen ( $5^{\text {th }}$ edition) were followed at all the steps. Semen samples were collected by masturbation in a private room near the laboratory, in order to limit the exposure of the semen to the fluctuations in temperature and to control the time between the collection and analysis. The samples were collected after a minimum of 2 days and a maximum of 7 days of sexual abstinence in a clean wide mouthed plastic container, which was confirmed to be nontoxic for spermatozoa. The specimen containers were kept at ambient temperature, between 20 degree Celsius and 37 degree Celsius to avoid large changes in temperature that may affect the spermatozoa after they were ejaculated into it. The report form of each patient was completed with the information of man's name, birth date, body mass index, the period of abstinence, date and time of semen collection and the interval between sample collection and start of semen analysis.

After initial macroscopic examination, the wet mount of the semen was seen under the inverted phase contrast microscope (Olympus). The microscope is equipped with $10 \mathrm{X}, 20 \mathrm{X}$ and 40X phase objectives for assessment of the sperm count, motility and vitality. It has a $100 \mathrm{X}$ oil immersion bright field objective for assessment of sperm morphology.

10 microlitres of the semen was placed on a microscope slide and was covered with $22 \mathrm{mmX} 22 \mathrm{~mm}$ cover slip. The sperm motility was assessed as soon as possible after liquefaction of the sample, preferably at 30 minutes, but in any case, within 1 hour following ejaculation to limit the deleterious effects of dehydration and changes in $\mathrm{pH}$ and temperature on motility. The spermatozoa in the area at least $5 \mathrm{~mm}$ from the edge of the coverslip were observed to avoid misinterpretations due to effects of drying on sperm motility. The sperm motility was expressed as progressive motility (PR), non-progressive motility (NP) and immotility (IM).

The sperm counting was done using 100 micrometer deep haemocytometer chambers. The dilution of semen required to allow sperm number to be measured accurately was assessed from an undiluted semen preparation. Sperm number was assessed in both the chambers of haemocytometer. If the two values agreed sufficiently, the aliquots taken were considered as representatives of sample.

The sperm morphology was studied by making smears of semen using feathering method. The Papanicolauo stain was used to assess the sperm morphology as its stains the acrosomal and post acrosomal regions of the head, excess residual cytoplasm, the mid piece and the principal piece. The slides stained using Pap procedure were permanently mounted and stored for future use in internal quality control programme (Figure 1).

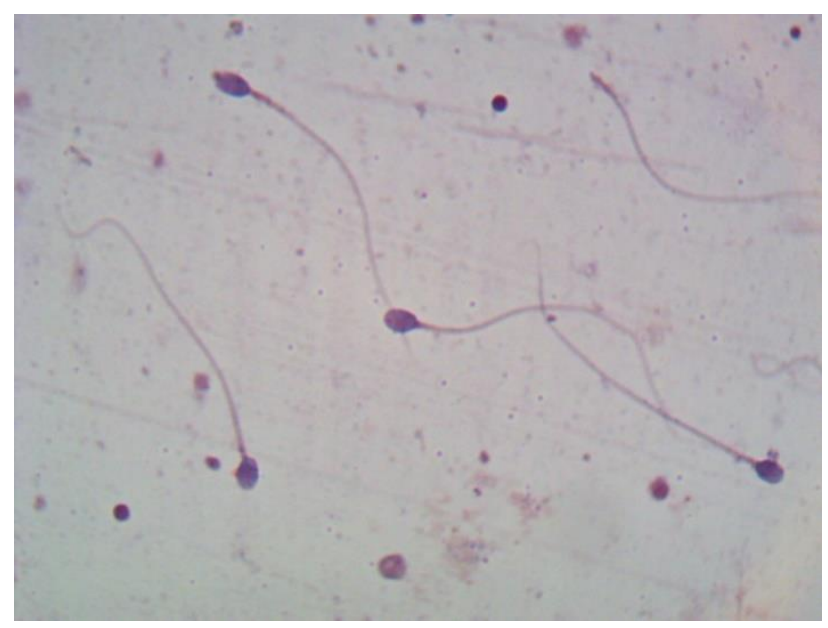

Figure 1: PAP stain for sperm morphology showing normal (centre) and abnormal spermatozoa with one pin head spermatozoon.

The morphological defects were classified and recorded meticulously. All the samples were screened for 200 sperms and in duplicate to prevent sampling errors. Agreement between replicates was determined for sperm numbers by the Poisson distribution and for percentages of motility and normal morphology by binomial distribution. 
The acceptable differences between the two percentages for a given average were based on the rounded $95 \%$ confidence interval. They were considered from the table provided in WHO manual for examination and processing of human semen ( $5^{\text {th }}$ edition). Larger than acceptable differences suggest the possibility of miscounting or errors of pipetting with nonrandom distribution of the sperms in the counting chamber.

Acidic Aniline Blue staining was used for DNA fragmentation index. The slides were prepared by feathering method and fixed in glutaraldehyde for half an hour. They were further stained by acidic aniline blue stain for 10 minutes. The slides were mounted and observed under oil immersion lens. The sperm heads with fragmented DNA were stained and appeared dark blue while the normal ones remained unstained (Figure 2). Two hundred sperms were observed and the DFI was recorded as percentage. ${ }^{7}$ The Acidic aniline blue method was validated by comparing the results with readymade kits like the Sperm halo test and acridine orange fluorescent staining methods.

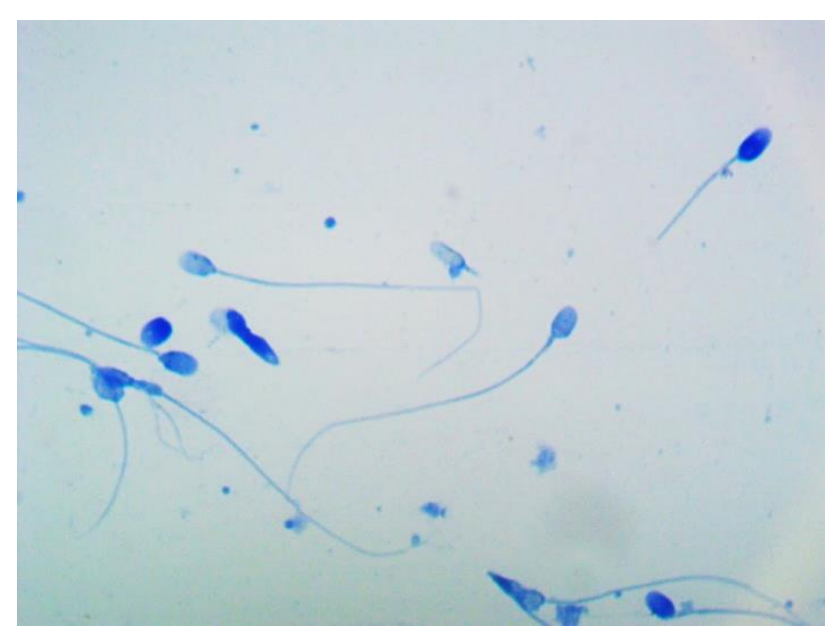

Figure 2: Acidic Aniline Blue staining for sperm chromatin - darkly stained sperm heads show abnormal sperm chromatin.

The results were compared by applying statistical tests as recommended by WHO 2010 manual. The stored semen samples (laboratory made) were used in IQC programme. The results were tabulated on daily basis by using a special software in an excel sheet. The statistical analysis was done with SPSS software using unpaired t-test. The sperm count, the percentage of progressive motility, percentage of normal forms and DFI were compared for the test of significance.

\section{RESULTS}

The total data studied was 4833 cases. The age wise distribution of cases was as shown in the Table 1.

The sperm parameters in the above three groups were studied. The average of sperm concentration, motility and
DFI with standard deviation was noted. It was seen that in younger age group the average sperm concentration was 34 million/ml while progressive motility was $38 \%$.

Table 1: Age wise distribution of cases.

\begin{tabular}{lll} 
Age & Nenumber of cases & $\%$ of cases \\
Group 1 $(<35)$ & 2615 & 54 \\
\hline Group 2 $(35-45)$ & 1899 & 39 \\
\hline Group 3 $(>45)$ & 319 & 7 \\
\hline Total & 4833 & 100 \\
\hline
\end{tabular}

The average of sperm concentration and progressive motility was maximum in group two with age between 35 to 45 years. The percentage of normal forms declined with increasing age as well as DFI values increased with increasing age, from $9.2 \pm 7.7$ in younger age group to $19.1 \pm 9.3$ in $>45$ years age group.

Table 2: Comparison of sperm parameters with DFI.

\begin{tabular}{lll} 
Parameters & $\begin{array}{l}\text { Group A } \\
(\text { DFI }<20)\end{array}$ & $\begin{array}{l}\text { Group B } \\
(\text { DFI }>20)\end{array}$ \\
\hline Sperm count & $35.21 \pm 14.3$ & $29.8 \pm 15.4$ \\
\hline Progressive motility & $38.2 \pm 12.4$ & $39.8 \pm 13.7$ \\
\hline Normal morphology & $9.6 \pm 8.4$ & $4.6 \pm 7.9$ \\
\hline Age & $35.6 \pm 42.6$ & $35.1 \pm 6.3$ \\
\hline Years of marriage & $7.2 \pm 5.3$ & $7.3 \pm 5.1$ \\
\hline
\end{tabular}

The average years of married life and presentation of the percentage of infertile males with respect to the years after marriage are shown in the Figure 3 . It was observed that $48 \%$ cases presented within 5 years of married life but almost $52 \%$ presented after 5 years of married life. The cases were divided according to the sperm concentration into four groups considering 15 million as cut off as per WHO guidelines.

Group I with sperm concentration less than 15 million, Group II with sperm concentration 15 to 30, Group III with sperm concentration 30 to 45 and Group IV having concentrations more than 45 million.

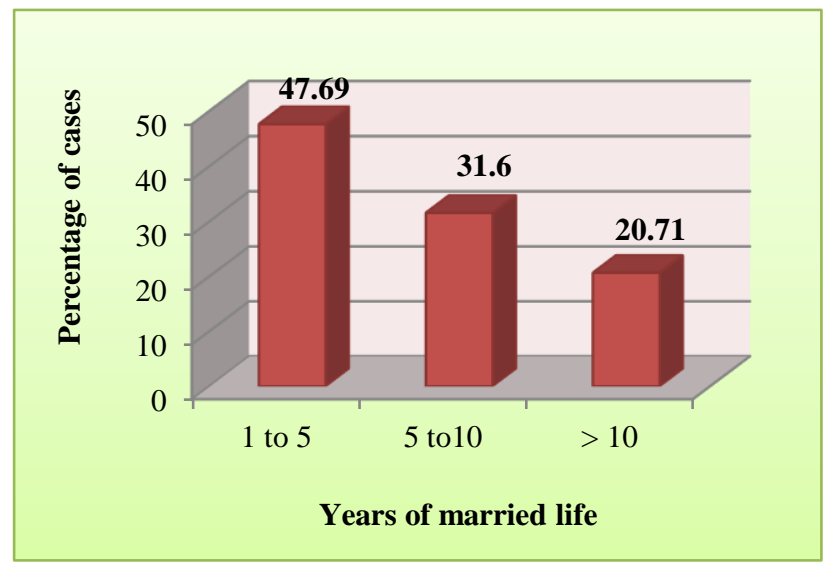

Figure 3: Presentation of infertile males with respect to years of married life. 
The distribution of cases in these four groups is as shown in Figure 4 and sperm parameters with $\mathrm{p}$ values in the groups were studied.

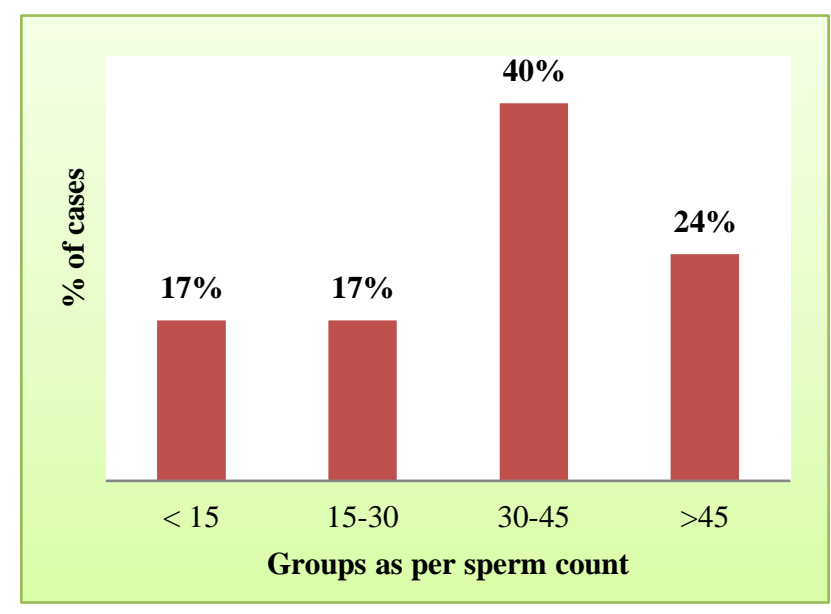

Figure 4: Distribution of cases as per sperm concentration.

The average sperm concentration in group I was 8.1 million/ml, $24.5 \%$ with progressive motility, $4.7 \%$ normal forms and an average DFI of $18.5 \%$. The average sperm concentration in group II was 26.5 million $/ \mathrm{ml}, 34.2 \%$ with progressive motility, $15.5 \%$ normal forms and an average DFI of $9.9 \%$.

The average sperm concentration in group III was 39.9 million/ml, $42.3 \%$ with progressive motility, $19.4 \%$ normal forms and an average DFI of $9.3 \%$. The average sperm concentration in group IV was $50.1 \mathrm{million} / \mathrm{ml}$, $44.1 \%$ with progressive motility, $12.7 \%$ normal forms and an average DFI of $8.1 \%$.

It was observed that the progressive motility, normal morphology and DFI showed statistically significant correlation with sperm count $(p$ value $=0.000)$. As the sperm concentration decreased the semen quality decreased while the DFI increased. Pearson correlation coefficient also showed positive correlation between sperm concentration, progressive motility and normal forms. The DFI showed negative correlation with sperm concentration; DFI increased with decreasing sperm concentration.

The cases were also divided according to their DFI to see the change in sperm parameters in patients with high DFI. Group A was having DFI less than 20 while group B had DFI more than or equal to 20 .

The parameters are shown in Table 2. There was statistically significant correlation ( $p$ value zero) between high DFI in group B and sperm concentration and morphology. However, motility and years of marriage had no correlation with high DFI. Though the DFI values increased with increasing age there was no statistically significant correlation with age and DFI.

\section{DISCUSSION}

The current study was conducted on 4833 infertile males. It holds importance because of the large sample size which is representative of the population. There are a very few studies in literature having such a large sample size.

In this study the age of the infertile males ranged between 22 to 55 years with maximum number of patients presenting between 25 to 45 years. The statistical analysis underlines the fact that $48 \%$ infertile males presented within five years of their married life while almost $52 \%$ presented late; after five years of marriage. In a study by Joshi et al published in 2011, the mean age of presentation was 20 to 35 years $(72 \%)$ while $66 \%$ patients presented within five years of marriage and $34 \%$ presented later than that. The late presentation could be because of the male dominated society where male factor for infertility is considered as a social taboo and is rarely discussed or accepted. ${ }^{6}$ Hence there is a need for increasing awareness about this fact in the society so that infertile couples could seek medical advice at the earliest.

The sperm parameters in the different age groups were studied. In the group with age between 35 to 45 years the sperm concentration, progressive motility and morphology were average and towards normal side. All parameters showed a decline as age advanced above 45 years. It is observed in different studies too that there is a decline in the sperm concentration with advancing age. It is therefore necessary that the male partner approaches for treatment at an early age. In developing countries, it is essential to create awareness about male infertility and motivate the male partner to come for early diagnosis and treatment. ${ }^{7}$ According to a study by Luna M, in 2009 advanced paternal age is inversely proportional to reproductive outcome. Hence, such efforts will help to improve the results of ART. ${ }^{8}$

According to WHO 2010 manual and other recent studies oligospermia is defined as concentration below 15 million. ${ }^{5}$ The sperm concentration was used to divide the infertile males into four groups. In the four groups it was seen that the percentage of cases in groups 1 and 2 were $17 \%$ each (total $34 \%$ ). It was seen that $40 \%$ of infertile males were in group 3 and had a sperm concentration between 30 to 45 million, while those with a concentration of more than 45 million were only $24 \%$. Global trends show that the sperm quality is deteriorating. There is overall decline in the sperm concentration, motility and morphology by $30 \%, 22.92 \%$ and $51.25 \%$ respectively. ${ }^{1,9}$ Authors tried to compare our findings with other studies which had a large sample size. Their results also confirmed an overall declining trend in sperm quality..$^{9-11}$ In a study conducted in Calcutta, semen samples from 3729 men were studied which showed a decline in sperm motility and seminal volume but no change in sperm concentration. ${ }^{12}$ Also a Danish study involving 4867 males carried out over a period of 15 years on sperm quality concluded that there 
is a decline in sperm concentration and morphology to affect the fertility of Danish males in coming future. ${ }^{13}$

An infertile couple is offered treatment in the form of many different assisted reproduction techniques [ART] such as intra-uterine insemination [IUI], in-vitro fertilization [IVF], intra-cytoplasmic sperm injection [ICSI] and many others. The success rates of ART procedures depend mainly on the quality and maturity of the gametes. Literature review indicates the importance of oligoasthenoteratozoospermia OAT and its impact on mode of ART, choice of ART and its outcome. ${ }^{14,15}$ The cases having OAT were better treated with ICSI, yet the success rates of ICSI were low. When sperm parameters were compared it was observed that there is a statistically significant difference in sperm parameters as the concentration increased from group 1 to 4 . These findings correlate with other studies where in some studies there was correlation between concentration and motility, while in others there was correlation between concentration and morphology. ${ }^{1,4,6,13}$ A recent study in 2017 by Kovac JR has stated that morphology by strict criteria should not be considered as the only parameter for ART or ICSI. It was seen in this study that sperms with zero normal forms conceived naturally. ${ }^{16}$

The DNA in the sperm nucleus plays a very important role and is called the sperm chromatin. This is assembled in a highly condensed form during spermiogenesis wherein the histones of the DNA get replaced through transition proteins. The compaction is done with the help of protamines, which now facilitates the faster movement of spermatozoon. Sperm chromatin compaction also plays a very important role in protecting the paternal genome and maintaining the integrity of sperm DNA. This nucleohistamine to nucleo-protamine transition plays a significant role in increasing the susceptibility of sperm DNA to injury. Such damaged or fragmented DNA is responsible for inability to fertilize or even if there is fertilization and pregnancy it may be short lived or lead to a fetus born with congenital anomalies. ${ }^{17,18}$ Literature search showed variability in the consensus on cut off values of DFI. In order to know the impact of DFI on sperm parameters and whether high DFI could show some pattern in sperm concentration and motility, we divided the samples into two groups.

The average sperm concentration and normal forms showed statistically significant difference between the two groups. While it was observed that there was no correlation between DFI and motility. Rather the results indicate that even if other sperm parameters may be normal, one has to be cautious about DFI. This is very important in cases where the sperm concentration is above 15 or 20 million, which is within the range of normalcy given by WHO and morphology is normal (as per WHO) but the patient is having failure of ART procedure. In such cases the DFI may be the cause for failure of pregnancy after embryo transfer leading to repeated abortions or failure to continue the pregnancy through term as also a cause for congenital anomalies. ${ }^{15-17,19}$ It was also observed that there was no significant correlation between DFI and age or years of married life of the male. However, in present study and study by Franco JG, the DFI increased with increasing age. ${ }^{17}$

From the above discussion it is clearly evident that sperm concentration is a good indicator of sperm quality. Further a good semen analysis report with emphasis on normal forms and DFI can clearly help the clinician to choose the treatment modality and counsel the couple regarding repeated pregnancy losses and any congenital fetal anomaly. In developing countries with increasing load of infertile patients and financial burden on the patients a simple test of basic semen analysis with sperm concentration, motility, morphology and sperm chromatin can be most rewarding.

\section{CONCLUSION}

In conclusion this study highlights the need for increasing awareness about male factor of infertility so that the infertile couple seeks help within first 3 to 4 years of married life when the semen and sperm parameters are of much better quality. A thorough semen analysis along with DFI can help the clinician to explain the couple in a better way about the choice of ART and success of treatment.

\section{ACKNOWLEDGMENTS}

Authors would like to thank supporting staff and colleagues and all the patients supported during study.

Funding: No funding sources

Conflict of interest: None declared

Ethical approval: The study was approved by the Institutional Ethics Committee

\section{REFERENCES}

1. Kumar N, Singh AK. Trends of male factor infertility, an important cause of infertility: a review of literature. J Hum Reprod Sci. 2015;8(4):191-6.

2. Kumar D. Prevalence of female infertility and its socio-economic factors in tribal communities of Central India. Rural Remote Health. 2007;7:456.

3. Zargar AH, Wani AI, Masoodi SR, Laway BA, Salahuddin M. Epidemiologic and etiologic aspects of primary infertility in the Kashmir region of India. Fertil Steril. 1997;68:637-43.

4. Ahmad MO, Khan SA, Hameed MA, Khan UA. A Study of Sperm Morphology in a Pakistani Population. Pak J Physiol. 2007;3(2).

5. World Health Organization. (2010). WHO laboratory manual for the examination and processing of human semen, $5^{\text {th }}$ ed. Geneva: World Health Organization Available http://www.who.int/iris/handle/10665/44261 
6. Clinical Atlas of Sperm Morphology: Achyut M. Phadke, First Edition, 2007, Jaypee Brothers, New Delhi, India.

7. Patil P, Bambulkar S, Ajgaonkar S, Patil R, Patil A, Nikam V. DNA fragmentation index [DFI] of human semen by modified aniline blue method. Cibtech $\mathrm{J}$ Bioprotocols. 2013;2(3):1-5.

8. Joshi P, Gopal N, Bhat V. Study of semen analysis patterns in infertile males. Int J Pharm Bio Sci. 2011 Jan;1(1):44-9.

9. Kovac JR, Addai J, Smith RP, Coward RM, Lamb DJ, Lipshultz LI. The effects of advanced paternal age on fertility. Asian J Andrology 2013;15(6):723-8.

10. Luna M, Finkler E, Barritt J, Barchama N, Sandler B, et al. Paternal age and assisted reproductive technology outcome in ovum recipients. Fertil Steril. 2009;92:1772-5.

11. Adiga SK, Jayaraman V, Kalthur G, Upadhya D, Kumar P. Declining semen quality among South Indian infertile men: a retrospective study. J Hum Reprod Sci. 2008;1:15-8.

12. Mukhopadhyay D, Varghese AC, Pal M, Banerjee SK, Bhattacharyya AK, Sharma RK, et al. Semen quality and age-specific changes: A study between two decades on 3,729 male partners of couples with normal sperm concentration and attending an andrology laboratory for infertility-related problems in an Indian city. Fertil Steril. 2010;93:2247-54.

13. Jørgensen N, Joensen UN, Jensen TK, et al. Human semen quality in the new millennium: a prospective cross-sectional population-based study of 4867 men. BMJ Open 2012;2:e000990.
14. Nikbakht R, Saharkhiz N. The influence of sperm morphology, total motile sperm concentration of semen and the number of motile sperm inseminated in sperm samples on the success of intrauterine insemination. Int J Fertil Steril. 2011;5(3):168-173.

15. Nordhoff V, Fricke RK, Schüring AN, Zitzmann M, Kliesch S. Treatment strategies for severe oligoasthenoteratozoospermia $\quad$ (OAT) $\quad(<0.1$ million/mL) patients. Andrology. 2015 Sep;3(5):85663.

16. Kovac JR, Smith RP, Cajipe M, Lamb DJ, Lipshultz LI. Men with a complete absence of normal sperm morphology exhibit high rates of success without assisted reproduction. Asian J Androl. 2017;19(1):3942.

17. Franco Jr JG. Sperm DNA fragmentation. Translational Androl Urol. 2017;16;6(4):S516-8.

18. Agarwal A, Majzoub A, Esteves SC, et al. Clinical utility of sperm DNA fragmentation testing: practice recommendations based on clinical scenarios. Transl Androl Urol. 2016;5:935-50.

19. Sakkas D, Alvarez JG. Sperm DNA fragmentation: mechanisms of origin, impact on reproductive outcome, and analysis. Fertil Steril. 2010;93:1027-36.

Cite this article as: Patil PS, Patki SM, Patki SS, Patki US. DNA fragmentation index and its correlation with other sperm parameters in 4833 infertile males. Int J Reprod Contracept Obstet Gynecol 2018;7:3718-23. 